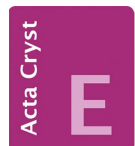

CRYSTALLOGRAPHIC COMMUNICATIONS

ISSN 2056-9890

Received 5 April 2016

Accepted 11 April 2016

Edited by $\mathrm{H}$. Stoeckli-Evans, University of Neuchâtel, Switzerland

Keywords: crystal structure; Brinzolamide; carbonic anhydrase inhibitor; sulfonamide; thiazine; absolute configuration; hydrogen bonding.

CCDC reference: 1473394

Supporting information: this article has supporting information at journals.iucr.org/e



OPEN $\odot$ ACCESS

\section{Crystal structure of Brinzolamide: a carbonic anhydrase inhibitor}

\author{
Huirong Zheng and Benyong Lou*
}

Department of Chemistry and Chemical Engineering, Minjiang University, Fuzhou 350108, People's Republic of China. *Correspondence e-mail: Iby@mju.edu.cn

In crystal structure of the title compound, $\mathrm{C}_{12} \mathrm{H}_{21} \mathrm{~N}_{3} \mathrm{O}_{5} \mathrm{~S}_{3}$ [systematic name: $(R)$ 4-ethylamino-2-(3-methoxypropyl)-3,4-dihydro- $2 H$-thieno[3,2-e][1,2] thiazine-6sulfonamide 1,1-dioxide], there exist three kinds of hydrogen-bonding interactions. The sulfonamide group is involved in hydrogen bonding with the secondary amine and the methoxy $\mathrm{O}$ atom, resulting in the formation of layers parallel to the $b c$ plane. The layers are linked by an $\mathrm{N}-\mathrm{H} \cdots \mathrm{O}$ hydrogen bond involving a sulfonamide $\mathrm{O}$ atom as acceptor and the secondary amine $\mathrm{H}$ atom as donor, which gives rise to the formation of a unique bilayer structure. The absolute structure of the molecule in the crystal was determined by resonant scattering [Flack parameter $=0.01(4)]$.

\section{Chemical context}

The crystal structures of organic solids are dominated mainly by hydrogen-bonding interactions (Steiner, 2002). Hydrogen bonding plays a crucial role in polymorphism of active pharmaceutical ingredients (Vippagunta et al., 2001). Brinzolamide (Conrow et al., 1999), is a carbonic anhydrase inhibitor used for the treatment of open-angle glaucoma or ocular hypertension (March \& Ochsner, 2000). Herein,we report on the crystal structure of Brinzolamide and the hydrogen-bonding interactions present in the crystal packing.<smiles>CCN[C@H]1CN(CCCOC)S(=O)(=O)c2sc(S(N)(=O)=O)cc21</smiles>

\section{Structural commentary}

The molecular structure of the title compound is shown in Fig. 1. The six-membered thiazine ring has an envelope conformation with the $\mathrm{N}$ atom, N2, as the flap. The 3-methoxypropyl chain has a twisted conformation with torsion angles $\mathrm{N} 2-\mathrm{C} 7-\mathrm{C} 8-\mathrm{C} 9, \mathrm{C} 7-\mathrm{C} 8-\mathrm{C} 9-\mathrm{O} 5$ and $\mathrm{C} 8-\mathrm{C} 9-$ O5-C10 being $71.66(18), 166.76(14)$ and $82.04(19)^{\circ}$, respectively. The ethylamino group $(\mathrm{N} 3 / \mathrm{C} 11 / \mathrm{C} 12)$ is normal to 


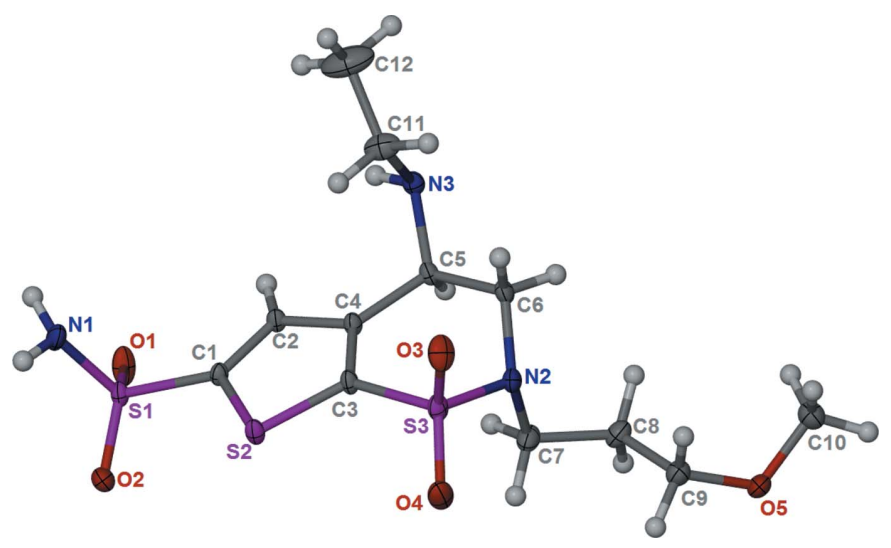

Figure 1

The molecular structure of the title compound, showing the atom labelling and $30 \%$ displacement ellipsoids.

the mean plane of the five planar atoms of the thiazine ring (S3/C3-C6), making a dihedral angle of $84.4(3)^{\circ}$. The three main functional groups (the sulfonamide, the secondary amine and the methoxy group) extend themselves in different directions, which facilitates the formation of a hydrogenbonded network.

\section{Supramolecular features}

There are three kinds of hydrogen-bonding interactions in the crystal of Brinzolamide (Table 1 and Figs. 2 and 3). The sulfonamide group is involved in hydrogen bonding [N1 ‥N3 $=2.886$ (2) $\AA$, Table 1] with the secondary amine, forming a $C(8)$ chain along the $b$-axis direction. The sulfonamide group is also involved in hydrogen bonding with the methoxy group
Table 1

Hydrogen-bond geometry $\left(\AA,^{\circ}\right)$.

\begin{tabular}{lllll}
\hline$D-\mathrm{H} \cdots A$ & $D-\mathrm{H}$ & $\mathrm{H} \cdots A$ & $D \cdots A$ & $D-\mathrm{H} \cdots A$ \\
\hline $\mathrm{N} 1-\mathrm{H} 1 B \cdots \mathrm{O}^{\mathrm{i}}$ & $0.87(1)$ & $1.98(1)$ & $2.841(2)$ & $177(2)$ \\
$\mathrm{N} 1-\mathrm{H} 1 A \cdots \mathrm{N3}^{\mathrm{ii}}$ & $0.87(1)$ & $2.03(1)$ & $2.886(2)$ & $171(2)$ \\
$\mathrm{N} 3-\mathrm{H} 3 \cdots \mathrm{O}^{\mathrm{iii}}$ & $0.86(1)$ & $2.26(1)$ & $3.042(2)$ & $151(2)$ \\
\hline
\end{tabular}

Symmetry codes: (i) $x, y+1, z-1$; (ii) $x, y+1, z$; (iii) $-x, y-\frac{1}{2},-z+1$.

[N1 $\cdots$ O5 = 2.841 (2) $\AA$, Table 1], linking the chains to form sheets parallel to the $b c$ plane (Fig. 2 and Table 1). There also exists another hydrogen bond between the sulfonamide and the secondary amine $[\mathrm{N} 3 \cdots \mathrm{O} 1=3.042$ (2) $\AA$, Table 1], linking the sheets to form a unique bilayer structure (Fig. 3).

\section{Database survey}

A search of the Cambridge Structural Database (CSD, Version 5.37, last update February 2016; Groom et al., 2016) revealed no hits for Brinzolamide. A search for the fused sixand five-membered ring system, viz. 3,4-dihydro- $2 \lambda^{2}$-thieno[3,2-e][1,2] thiazine 1,1-dioxide, gave only two hits: 8 b-bromo2-(bromomethyl)-4-methyl-3a-phenyl-1,3a,4,8b-tetrahydro$2 H$-furo[2,3-c]thieno[3,2-e][1,2]thiazine 5,5-dioxide (BUFQIE; Barange et al., 2014) and (S)-6,6-dimethyl-4a,5,6,7tetrahydro-4H-pyrrolo[1,2-b] thieno[3,2-e][1,2]thiazine 9,9dioxide (BUXDEE; Zeng \& Chemler, 2007). The latter crystallizes in the chiral monoclinic space group $P 2_{1}$, with four independent molecules in the asymmetric unit. However, in both compounds the six-membered thiazine ring is also fused to a second five-membered ring; a tetrahydrofuro ring in the case of BUFQIE, fused to the $\mathrm{C}-\mathrm{C}$ bond, and a pyrrolo ring

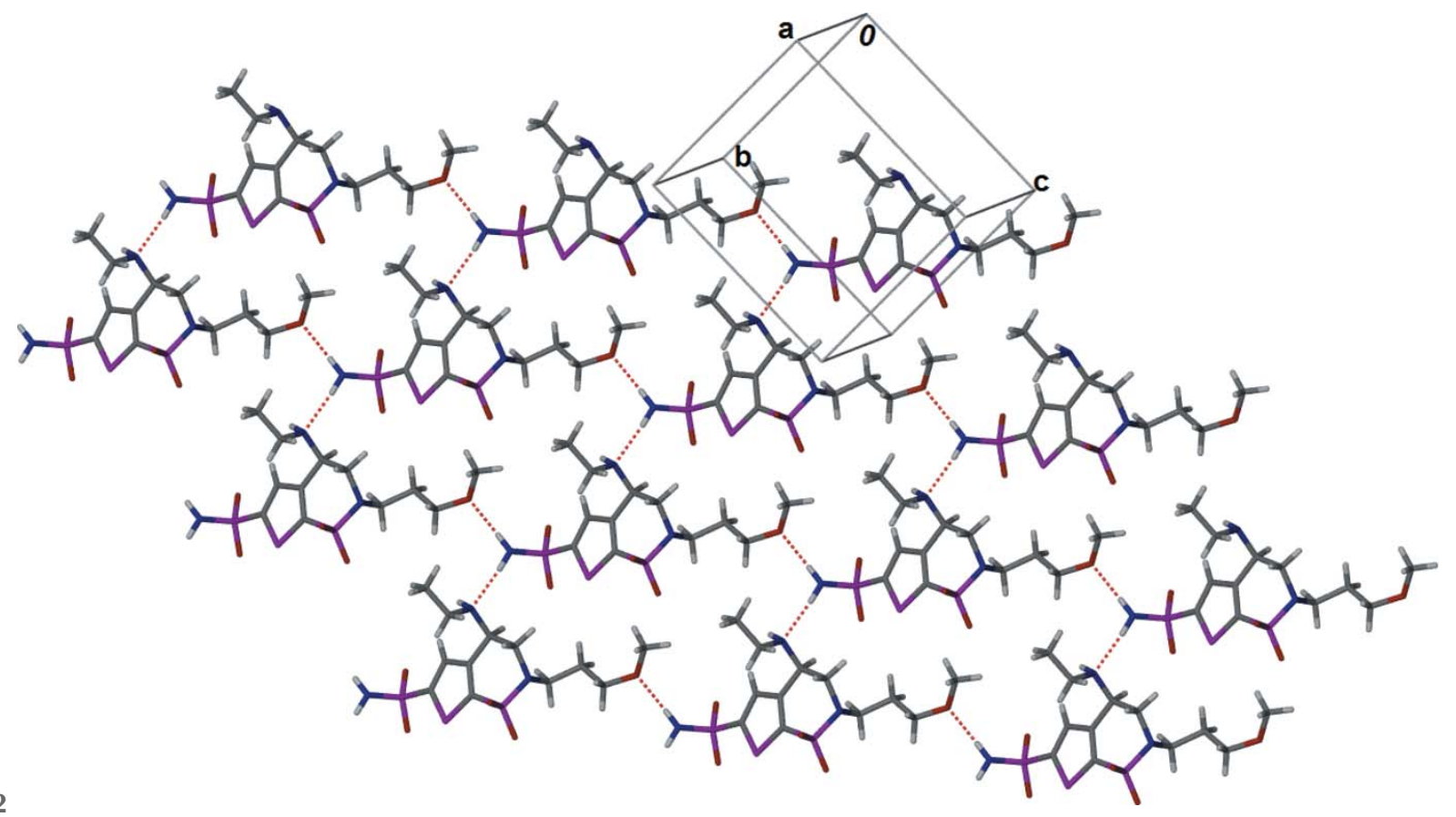

Figure 2

A view along the $a$ axis of the two-dimensional hydrogen-bonded network in the crystal of the title compound. The hydrogen bonds are shown as dashed lines (see Table 1 for details). 
in the case of BUXDEE, fused to the $\mathrm{N}-\mathrm{C}$ bond. The thiazine ring in BUFQIE has a distorted twist-boat conformation, while in BUFQIE all four independent molecules have halfchair conformations. This is in contrast to the situation in the title compound where the thiazine ring has an envelope conformation with the $\mathrm{N}$ atom as the flap.

\section{Synthesis and crystallization}

The enantioselective synthesis of Brinzolamide has been reported by Conrow et al., (1999). It is marketed under the trade name of Azopt by Alcon Laboratories, Inc., Fort Worth, Texas 76134, USA. Colourless prismatic crystals of Brinzolamide (383 mg, $1 \mathrm{mmol}$ ) were obtained by slow evaporation of a solution in chloroform $(15 \mathrm{ml})$.

\section{Refinement}

Crystal data, data collection and structure refinement details are summarized in Table 2. The $\mathrm{NH}$ and $\mathrm{NH}_{2} \mathrm{H}$ atoms were located in difference Fourier maps and refined with distance restraints of $\mathrm{N}-\mathrm{H}=0.87$ (1) $\AA$ for $\mathrm{NH}$ and 0.86 (1) $\AA$ for $\mathrm{NH}_{2}$ $\mathrm{H}$ atoms. The $\mathrm{C}$-bound $\mathrm{H}$ atoms were included in calculated positions and treated as riding atoms: $\mathrm{C}-\mathrm{H}=0.95-1.00 \AA$ with $U_{\text {iso }}(\mathrm{H})=1.5 U_{\text {eq }}(\mathrm{C}$-methyl $)$ and $1.2 U_{\text {eq }}(\mathrm{C})$ for other $\mathrm{H}$ atoms. The absolute structure of the molecule in the crystal was determined by resonant scattering [Flack parameter = $0.01(4)]$.
Table 2

Experimental details.

\begin{tabular}{|c|c|}
\hline \multicolumn{2}{|l|}{ Crystal data } \\
\hline Chemical formula & $\mathrm{C}_{12} \mathrm{H}_{21} \mathrm{~N}_{3} \mathrm{O}_{5} \mathrm{~S}_{3}$ \\
\hline$M_{\mathrm{r}}$ & 383.50 \\
\hline Crystal system, space group & Monoclinic, $P 2_{1}$ \\
\hline Temperature $(\mathrm{K})$ & 293 \\
\hline$a, b, c(\AA)$ & $9.698(2), 8.8127(19), 10.133$ (2) \\
\hline$\beta\left(^{\circ}\right)$ & $92.248(3)$ \\
\hline$V\left(\AA^{3}\right)$ & $865.4(3)$ \\
\hline$Z$ & 2 \\
\hline Radiation type & Mo $K \alpha$ \\
\hline$\mu\left(\mathrm{mm}^{-1}\right)$ & 0.46 \\
\hline Crystal size $(\mathrm{mm})$ & $0.35 \times 0.35 \times 0.20$ \\
\hline \multicolumn{2}{|l|}{ Data collection } \\
\hline Diffractometer & Rigaku Mercury CCD \\
\hline Absorption correction & $\begin{array}{l}\text { Multi-scan (CrystalClear; Rigaku, } \\
\text { 2000) }\end{array}$ \\
\hline$T_{\min }, T_{\max }$ & $0.853,0.913$ \\
\hline $\begin{array}{l}\text { No. of measured, independent and } \\
\text { observed }[I>2 \sigma(I)] \text { reflections }\end{array}$ & $6608,3684,3612$ \\
\hline$R_{\text {int }}$ & 0.010 \\
\hline$(\sin \theta / \lambda)_{\max }\left(\AA^{-1}\right)$ & 0.649 \\
\hline \multicolumn{2}{|l|}{ Refinement } \\
\hline$R\left[F^{2}>2 \sigma\left(F^{2}\right)\right], w R\left(F^{2}\right), S$ & $0.022,0.059,1.04$ \\
\hline No. of reflections & 3684 \\
\hline No. of parameters & 222 \\
\hline No. of restraints & 4 \\
\hline $\mathrm{H}$-atom treatment & $\begin{array}{l}\mathrm{H} \text { atoms treated by a mixture of } \\
\text { independent and constrained } \\
\text { refinement }\end{array}$ \\
\hline$\Delta \rho_{\max }, \Delta \rho_{\min }\left(\mathrm{e} \AA^{-3}\right)$ & $0.21,-0.19$ \\
\hline Absolute structure & 1595 Friedel pairs; Flack (1983) \\
\hline Absolute structure parameter & $0.01(4)$ \\
\hline
\end{tabular}

Computer programs: CrystalClear (Rigaku, 2000), SHELXS97 and SHELXL97 (Sheldrick, 2008), X-SEED (Barbour, 2001) and PLATON (Spek, 2009).

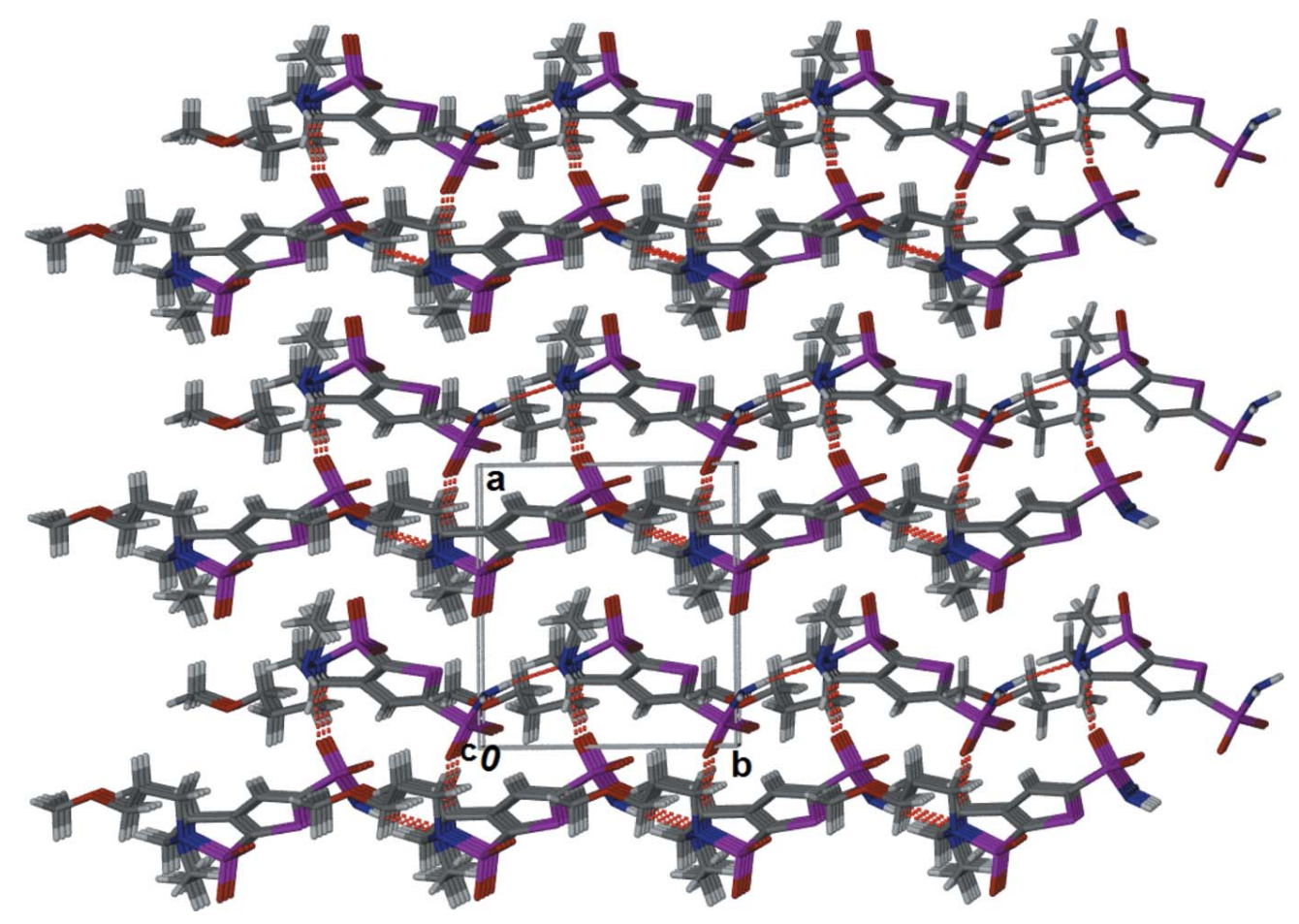

Figure 3

A view along the $c$ axis of the crystal packing of the title compound, showing the hydrogen bonded bilayer structure. The hydrogen bonds are shown as dashed lines (see Table 1 for details). 


\section{Acknowledgements}

The authors are grateful for a grant (No. 2015 J01599) from the Natural Science Foundation of Fujian Province.

\section{References}

Barange, D. K., Kavala, V., Kuo, C.-W., Wang, C.-C., Rajawinslin, R. R., Donala, J. \& Yao, C.-F. (2014). Tetrahedron, 70, 7598-7605. Barbour, L. J. (2001). J. Supramol. Chem. 1, 189-191.

Conrow, R. E., Dean, W. D., Zinke, P. W., Deason, M. E., Sproull, S. J., Dantanarayana, A. P. \& DuPriest, M. T. (1999). Org. Process Res. Dev. 3, 114-120.
Flack, H. D. (1983). Acta Cryst. A39, 876-881.

Groom, C. R., Bruno, I. J., Lightfoot, M. P. \& Ward, S. C. (2016). Acta Cryst. B72, 171-179.

March, W. F. \& Ochsner, K. I. (2000). Am. J. Ophthalmol. 129, 136143.

Rigaku (2000). CrystalClear. Rigaku Corporation, Tokyo, Japan.

Sheldrick, G. M. (2008). Acta Cryst. A64, 112-122.

Spek, A. L. (2009). Acta Cryst. D65, 148-155.

Steiner, T. (2002). Angew. Chem. Int. Ed. 41, 48-76.

Vippagunta, S. R., Brittain, H. G. \& Grant, D. J. W. (2001). Adv. Drug Deliv. Rev. 48, 3-26.

Zeng, W. \& Chemler, S. R. (2007). J. Am. Chem. Soc. 129, 1294812949. 


\section{supporting information}

Acta Cryst. (2016). E72, 692-695 [doi:10.1107/S2056989016006022]

\section{Crystal structure of Brinzolamide: a carbonic anhydrase inhibitor}

\section{Huirong Zheng and Benyong Lou}

Computing details

Data collection: CrystalClear (Rigaku, 2000); cell refinement: CrystalClear (Rigaku, 2000); data reduction: CrystalClear (Rigaku, 2000); program(s) used to solve structure: SHELXS97 (Sheldrick, 2008); program(s) used to refine structure: SHELXL97 (Sheldrick, 2008); molecular graphics: X-SEED (Barbour, 2001); software used to prepare material for publication: SHELXL97 (Sheldrick, 2008) and PLATON (Spek, 2009).

(5R)-5-ethylamino-3-(3-methoxypropyl)-2,2-dioxo-2,9-dithia-3-azabicyclo[4.3.0] nona-1 (6)7-diene-8sulfonamide

Crystal data

$\mathrm{C}_{12} \mathrm{H}_{21} \mathrm{~N}_{3} \mathrm{O}_{5} \mathrm{~S}_{3}$

$M_{r}=383.50$

Monoclinic, $P 2_{1}$

Hall symbol: $\mathrm{P} 2 \mathrm{yb}$

$a=9.698(2) \AA$

$b=8.8127(19) \AA$

$c=10.133(2) \AA$

$\beta=92.248(3)^{\circ}$

$V=865.4(3) \AA^{3}$

$Z=2$

Data collection

Rigaku Mercury CCD diffractometer

Radiation source: fine-focus sealed tube

Graphite monochromator

Detector resolution: 14.6306 pixels $\mathrm{mm}^{-1}$

CCD_Profile_fitting scans

Absorption correction: multi-scan

(CrystalClear; Rigaku, 2000)

$T_{\min }=0.853, T_{\max }=0.913$

Refinement

Refinement on $F^{2}$

Least-squares matrix: full

$R\left[F^{2}>2 \sigma\left(F^{2}\right)\right]=0.022$

$w R\left(F^{2}\right)=0.059$

$S=1.03$

3684 reflections

222 parameters

4 restraints
$F(000)=404$

$D_{\mathrm{x}}=1.472 \mathrm{Mg} \mathrm{m}^{-3}$

Mo $K \alpha$ radiation, $\lambda=0.71073 \AA$

Cell parameters from 2619 reflections

$\theta=2.1-27.5^{\circ}$

$\mu=0.46 \mathrm{~mm}^{-1}$

$T=293 \mathrm{~K}$

Prism, colourless

$0.35 \times 0.35 \times 0.20 \mathrm{~mm}$

6608 measured reflections

3684 independent reflections

3612 reflections with $I>2 \sigma(I)$

$R_{\text {int }}=0.010$

$\theta_{\text {max }}=27.5^{\circ}, \theta_{\text {min }}=2.0^{\circ}$

$h=-12 \rightarrow 12$

$k=-11 \rightarrow 11$

$l=-13 \rightarrow 13$

Primary atom site location: structure-invariant direct methods

Secondary atom site location: difference Fourier map

Hydrogen site location: inferred from neighbouring sites

$\mathrm{H}$ atoms treated by a mixture of independent and constrained refinement 
$w=1 /\left[\sigma^{2}\left(F_{\mathrm{o}}^{2}\right)+(0.0403 P)^{2}+0.0611 P\right]$

where $P=\left(F_{\mathrm{o}}^{2}+2 F_{\mathrm{c}}^{2}\right) / 3$

$(\Delta / \sigma)_{\max }=0.001$

$\Delta \rho_{\max }=0.21$ e $\AA^{-3}$
$\Delta \rho_{\min }=-0.19$ e $\AA^{-3}$

Absolute structure: 1595 Friedel pairs; Flack (1983)

Absolute structure parameter: $0.01(4)$

\section{Special details}

Geometry. All esds (except the esd in the dihedral angle between two 1.s. planes) are estimated using the full covariance matrix. The cell esds are taken into account individually in the estimation of esds in distances, angles and torsion angles; correlations between esds in cell parameters are only used when they are defined by crystal symmetry. An approximate (isotropic) treatment of cell esds is used for estimating esds involving l.s. planes.

Refinement. Refinement of $\mathrm{F}^{2}$ against ALL reflections. The weighted R-factor $\mathrm{wR}$ and goodness of fit $\mathrm{S}$ are based on $\mathrm{F}^{2}$, conventional $R$-factors $R$ are based on $F$, with $F$ set to zero for negative $F^{2}$. The threshold expression of $F^{2}>2 \operatorname{sigma}\left(\mathrm{F}^{2}\right)$ is used only for calculating R-factors(gt) etc. and is not relevant to the choice of reflections for refinement. R-factors based on $\mathrm{F}^{2}$ are statistically about twice as large as those based on F, and R- factors based on ALL data will be even larger.

Fractional atomic coordinates and isotropic or equivalent isotropic displacement parameters $\left(\AA^{2}\right)$

\begin{tabular}{|c|c|c|c|c|}
\hline & $x$ & $y$ & $z$ & $U_{\text {iso }} * / U_{\text {eq }}$ \\
\hline $\mathrm{S} 1$ & $0.08557(3)$ & $0.95125(4)$ & $0.63688(3)$ & $0.02705(9)$ \\
\hline $\mathrm{S} 2$ & $0.26509(4)$ & $0.81437(4)$ & $0.85525(3)$ & $0.02964(9)$ \\
\hline $\mathrm{S} 3$ & $0.37674(4)$ & $0.52417(4)$ & $0.99143(4)$ & $0.03230(9)$ \\
\hline $\mathrm{O} 1$ & $-0.02692(13)$ & $0.88932(16)$ & $0.55817(13)$ & $0.0482(3)$ \\
\hline $\mathrm{O} 2$ & 0.05935 (13) & $1.05211(14)$ & $0.74416(12)$ & $0.0409(3)$ \\
\hline $\mathrm{O} 3$ & $0.51842(12)$ & $0.50532(17)$ & $0.96186(14)$ & $0.0482(3)$ \\
\hline $\mathrm{O} 4$ & $0.34354(16)$ & $0.59370(17)$ & $1.11319(11)$ & $0.0504(3)$ \\
\hline O5 & $0.14543(15)$ & $0.01552(16)$ & $1.26375(12)$ & $0.0472(3)$ \\
\hline N1 & $0.19095(16)$ & $1.02862(18)$ & $0.54219(13)$ & 0.0365 \\
\hline N2 & $0.30128(13)$ & $0.35864(15)$ & $0.97875(12)$ & 0.0310 \\
\hline N3 & $0.29017(12)$ & $0.32938(16)$ & $0.60656(12)$ & 0.0285 \\
\hline $\mathrm{C} 1$ & $0.17016(14)$ & $0.79352(17)$ & $0.70936(12)$ & $0.0241(3)$ \\
\hline $\mathrm{C} 2$ & $0.16170(15)$ & $0.64755(17)$ & $0.66712(13)$ & $0.0262(3)$ \\
\hline $\mathrm{H} 2$ & 0.1130 & 0.6168 & 0.5884 & $0.031 *$ \\
\hline $\mathrm{C} 3$ & $0.29337(15)$ & $0.62151(18)$ & $0.85939(14)$ & $0.0269(3)$ \\
\hline $\mathrm{C} 4$ & $0.23410(14)$ & $0.54569(18)$ & $0.75421(13)$ & $0.0248(3)$ \\
\hline $\mathrm{C} 5$ & $0.23691(15)$ & $0.37508(18)$ & $0.73479(14)$ & $0.0262(3)$ \\
\hline H5 & 0.1394 & 0.3386 & 0.7368 & $0.031^{*}$ \\
\hline C6 & $0.31904(17)$ & $0.29191(18)$ & $0.84604(15)$ & $0.0312(3)$ \\
\hline H6A & 0.2895 & 0.1844 & 0.8473 & $0.037 *$ \\
\hline H6B & 0.4182 & 0.2939 & 0.8264 & $0.037 *$ \\
\hline $\mathrm{C} 7$ & $0.15834(18)$ & $0.3485(2)$ & $1.02768(17)$ & $0.0410(4)$ \\
\hline H7A & 0.0916 & 0.3777 & 0.9557 & $0.049 *$ \\
\hline H7B & 0.1486 & 0.4212 & 1.1012 & $0.049 *$ \\
\hline $\mathrm{C} 8$ & 0.12434 (19) & $0.1893(2)$ & $1.07530(16)$ & 0.0409 (4) \\
\hline H8A & 0.0237 & 0.1818 & 1.0872 & $0.049 *$ \\
\hline H8B & 0.1489 & 0.1149 & 1.0069 & $0.049^{*}$ \\
\hline $\mathrm{C} 9$ & $0.2000(2)$ & $0.1493(2)$ & $1.20395(16)$ & $0.0414(4)$ \\
\hline H9A & 0.2988 & 0.1329 & 1.1873 & $0.050 *$ \\
\hline H9B & 0.1935 & 0.2355 & 1.2661 & $0.050 *$ \\
\hline $\mathrm{C} 10$ & $0.1941(2)$ & -0.1233 & $1.2128(2)$ & $0.0497(5)$ \\
\hline
\end{tabular}




\begin{tabular}{lllll} 
H10A & 0.1556 & -0.1381 & 1.1229 & $0.074^{*}$ \\
H10B & 0.1652 & -0.2072 & 1.2690 & $0.074^{*}$ \\
H10C & 0.2950 & -0.1209 & 1.2114 & $0.074^{*}$ \\
C11 & $0.42904(19)$ & $0.3825(3)$ & $0.57763(18)$ & $0.0499(5)$ \\
H11A & 0.4982 & 0.3234 & 0.6305 & $0.060^{*}$ \\
H11B & 0.4386 & 0.4905 & 0.6033 & $0.060^{*}$ \\
C12 & $0.4563(3)$ & $0.3657(5)$ & $0.4339(2)$ & $0.0923(12)$ \\
H12A & 0.4480 & 0.2586 & 0.4087 & $0.138^{*}$ \\
H12B & 0.5497 & 0.4016 & 0.4175 & $0.138^{*}$ \\
H12C & 0.3890 & 0.4258 & 0.3816 & $0.138^{*}$ \\
H3 & $0.2359(17)$ & $0.369(2)$ & $0.5477(16)$ & $0.038(5)^{*}$ \\
H1A & $0.228(2)$ & $1.1141(16)$ & $0.566(2)$ & $0.044(6)^{*}$ \\
H1B & $0.174(2)$ & $1.024(3)$ & $0.4577(10)$ & $0.046(5)^{*}$ \\
\hline
\end{tabular}

Atomic displacement parameters $\left(\AA^{2}\right)$

\begin{tabular}{lllllll}
\hline & $U^{11}$ & $U^{22}$ & $U^{33}$ & $U^{12}$ & $U^{13}$ & $U^{23}$ \\
\hline S1 & $0.03112(17)$ & $0.02019(17)$ & $0.02929(17)$ & $0.00014(14)$ & $-0.00565(13)$ & $0.00508(14)$ \\
S2 & $0.04226(19)$ & $0.01977(18)$ & $0.02583(16)$ & $-0.00143(14)$ & $-0.01218(13)$ & $-0.00112(14)$ \\
S3 & $0.0398(2)$ & $0.0277(2)$ & $0.02842(17)$ & $0.00293(16)$ & $-0.01163(13)$ & $0.00436(15)$ \\
O1 & $0.0434(7)$ & $0.0388(7)$ & $0.0599(8)$ & $-0.0078(6)$ & $-0.0284(6)$ & $0.0154(6)$ \\
O2 & $0.0534(7)$ & $0.0300(7)$ & $0.0398(6)$ & $0.0094(5)$ & $0.0096(5)$ & $0.0022(5)$ \\
O3 & $0.0345(6)$ & $0.0476(8)$ & $0.0612(8)$ & $0.0005(6)$ & $-0.0145(5)$ & $0.0135(7)$ \\
O4 & $0.0838(10)$ & $0.0383(7)$ & $0.0280(6)$ & $0.0073(7)$ & $-0.0134(6)$ & $-0.0016(5)$ \\
O5 & $0.0716(9)$ & $0.0387(7)$ & $0.0326(6)$ & $0.0081(7)$ & $0.0188(6)$ & $0.0074(6)$ \\
N1 & $0.0563(8)$ & $0.0259(8)$ & $0.0274(6)$ & $-0.0088(7)$ & $0.0012(5)$ & $0.0046(6)$ \\
N2 & $0.0382(6)$ & $0.0250(7)$ & $0.0297(6)$ & $0.0047(5)$ & $0.0010(5)$ & $0.0062(5)$ \\
N3 & $0.0306(6)$ & $0.0254(7)$ & $0.0291(6)$ & $-0.0013(5)$ & $-0.0040(4)$ & $-0.0020(5)$ \\
C1 & $0.0283(6)$ & $0.0219(8)$ & $0.0216(6)$ & $-0.0007(5)$ & $-0.0055(5)$ & $0.0033(5)$ \\
C2 & $0.0324(7)$ & $0.0211(7)$ & $0.0246(6)$ & $-0.0015(5)$ & $-0.0064(5)$ & $0.0004(6)$ \\
C3 & $0.0328(7)$ & $0.0196(8)$ & $0.0276(7)$ & $0.0021(6)$ & $-0.0074(5)$ & $0.0031(6)$ \\
C4 & $0.0279(6)$ & $0.0205(7)$ & $0.0257(6)$ & $0.0010(5)$ & $-0.0037(5)$ & $0.0025(6)$ \\
C5 & $0.0280(6)$ & $0.0202(7)$ & $0.0301(7)$ & $0.0013(5)$ & $-0.0034(5)$ & $0.0005(6)$ \\
C6 & $0.0394(7)$ & $0.0217(8)$ & $0.0322(7)$ & $0.0059(6)$ & $-0.0008(6)$ & $0.0026(6)$ \\
C7 & $0.0414(8)$ & $0.0419(11)$ & $0.0402(8)$ & $0.0084(7)$ & $0.0087(6)$ & $0.0116(7)$ \\
C8 & $0.0458(9)$ & $0.0453(11)$ & $0.0317(7)$ & $-0.0054(8)$ & $0.0035(6)$ & $0.0066(8)$ \\
C9 & $0.0549(10)$ & $0.0368(10)$ & $0.0327(8)$ & $0.0009(8)$ & $0.0042(7)$ & $0.0026(7)$ \\
C10 & $0.0651(12)$ & $0.0380(10)$ & $0.0462(10)$ & $0.0054(9)$ & $0.0051(8)$ & $-0.0013(9)$ \\
C11 & $0.0390(9)$ & $0.0666(13)$ & $0.0444(9)$ & $-0.0152(9)$ & $0.0070(7)$ & $-0.0117(10)$ \\
C12 & $0.0653(15)$ & $0.153(4)$ & $0.0599(14)$ & $-0.0316(19)$ & $0.0227(11)$ & $-0.0246(18)$ \\
& & & & & & \\
\hline
\end{tabular}

Geometric parameters $\left(\AA,{ }^{\circ}\right)$

\begin{tabular}{llll}
\hline $\mathrm{S} 1-\mathrm{O} 1$ & $1.4338(12)$ & $\mathrm{C} 4-\mathrm{C} 5$ & $1.517(2)$ \\
$\mathrm{S} 1-\mathrm{O} 2$ & $1.4346(13)$ & $\mathrm{C} 5-\mathrm{C} 6$ & $1.540(2)$ \\
$\mathrm{S} 1-\mathrm{N} 1$ & $1.5834(14)$ & $\mathrm{C} 5-\mathrm{H} 5$ & 1.0000 \\
$\mathrm{~S} 1-\mathrm{C} 1$ & $1.7600(15)$ & $\mathrm{C} 6-\mathrm{H} 6 \mathrm{~A}$ & 0.9900 \\
$\mathrm{~S} 2-\mathrm{C} 1$ & $1.7205(13)$ & $\mathrm{C} 6-\mathrm{H} 6 \mathrm{~B}$ & 0.9900
\end{tabular}




\begin{tabular}{|c|c|c|c|}
\hline $\mathrm{S} 2-\mathrm{C} 3$ & $1.7219(16)$ & $\mathrm{C} 7-\mathrm{C} 8$ & $1.524(3)$ \\
\hline $\mathrm{S} 3-\mathrm{O} 4$ & $1.4256(14)$ & $\mathrm{C} 7-\mathrm{H} 7 \mathrm{~A}$ & 0.9900 \\
\hline $\mathrm{S} 3-\mathrm{O} 3$ & $1.4274(14)$ & $\mathrm{C} 7-\mathrm{H} 7 \mathrm{~B}$ & 0.9900 \\
\hline $\mathrm{S} 3-\mathrm{N} 2$ & $1.6349(15)$ & $\mathrm{C} 8-\mathrm{C} 9$ & $1.513(2)$ \\
\hline $\mathrm{S} 3-\mathrm{C} 3$ & $1.7592(14)$ & $\mathrm{C} 8-\mathrm{H} 8 \mathrm{~A}$ & 0.9900 \\
\hline $\mathrm{O} 5-\mathrm{C} 10$ & $1.416(2)$ & $\mathrm{C} 8-\mathrm{H} 8 \mathrm{~B}$ & 0.9900 \\
\hline $\mathrm{O} 5-\mathrm{C} 9$ & $1.436(2)$ & $\mathrm{C} 9-\mathrm{H} 9 \mathrm{~A}$ & 0.9900 \\
\hline N1-H1A & $0.866(10)$ & C9-H9B & 0.9900 \\
\hline N1-H1B & $0.866(9)$ & $\mathrm{C} 10-\mathrm{H} 10 \mathrm{~A}$ & 0.9800 \\
\hline $\mathrm{N} 2-\mathrm{C} 6$ & $1.484(2)$ & $\mathrm{C} 10-\mathrm{H} 10 \mathrm{~B}$ & 0.9800 \\
\hline $\mathrm{N} 2-\mathrm{C} 7$ & $1.493(2)$ & $\mathrm{C} 10-\mathrm{H} 10 \mathrm{C}$ & 0.9800 \\
\hline $\mathrm{N} 3-\mathrm{C} 11$ & $1.466(2)$ & $\mathrm{C} 11-\mathrm{C} 12$ & $1.497(3)$ \\
\hline $\mathrm{N} 3-\mathrm{C} 5$ & $1.4731(19)$ & C11-H11A & 0.9900 \\
\hline $\mathrm{N} 3-\mathrm{H} 3$ & $0.856(9)$ & C11-H11B & 0.9900 \\
\hline $\mathrm{C} 1-\mathrm{C} 2$ & $1.357(2)$ & $\mathrm{C} 12-\mathrm{H} 12 \mathrm{~A}$ & 0.9800 \\
\hline $\mathrm{C} 2-\mathrm{C} 4$ & $1.4245(19)$ & $\mathrm{C} 12-\mathrm{H} 12 \mathrm{~B}$ & 0.9800 \\
\hline $\mathrm{C} 2-\mathrm{H} 2$ & 0.9500 & $\mathrm{C} 12-\mathrm{H} 12 \mathrm{C}$ & 0.9800 \\
\hline $\mathrm{C} 3-\mathrm{C} 4$ & $1.365(2)$ & & \\
\hline $\mathrm{O} 1-\mathrm{S} 1-\mathrm{O} 2$ & $120.25(9)$ & $\mathrm{N} 2-\mathrm{C} 6-\mathrm{H} 6 \mathrm{~A}$ & 108.9 \\
\hline $\mathrm{O} 1-\mathrm{S} 1-\mathrm{N} 1$ & $108.78(8)$ & $\mathrm{C} 5-\mathrm{C} 6-\mathrm{H} 6 \mathrm{~A}$ & 108.9 \\
\hline $\mathrm{O} 2-\mathrm{S} 1-\mathrm{N} 1$ & $109.28(8)$ & $\mathrm{N} 2-\mathrm{C} 6-\mathrm{H} 6 \mathrm{~B}$ & 108.9 \\
\hline $\mathrm{O} 1-\mathrm{S} 1-\mathrm{C} 1$ & $105.28(7)$ & $\mathrm{C} 5-\mathrm{C} 6-\mathrm{H} 6 \mathrm{~B}$ & 108.9 \\
\hline $\mathrm{O} 2-\mathrm{S} 1-\mathrm{C} 1$ & $105.47(7)$ & $\mathrm{H} 6 \mathrm{~A}-\mathrm{C} 6-\mathrm{H} 6 \mathrm{~B}$ & 107.7 \\
\hline $\mathrm{N} 1-\mathrm{S} 1-\mathrm{C} 1$ & $106.95(8)$ & $\mathrm{N} 2-\mathrm{C} 7-\mathrm{C} 8$ & $112.05(14)$ \\
\hline $\mathrm{C} 1-\mathrm{S} 2-\mathrm{C} 3$ & $89.70(7)$ & $\mathrm{N} 2-\mathrm{C} 7-\mathrm{H} 7 \mathrm{~A}$ & 109.2 \\
\hline $\mathrm{O} 4-\mathrm{S} 3-\mathrm{O} 3$ & $118.92(9)$ & $\mathrm{C} 8-\mathrm{C} 7-\mathrm{H} 7 \mathrm{~A}$ & 109.2 \\
\hline $\mathrm{O} 4-\mathrm{S} 3-\mathrm{N} 2$ & $109.63(8)$ & $\mathrm{N} 2-\mathrm{C} 7-\mathrm{H} 7 \mathrm{~B}$ & 109.2 \\
\hline $\mathrm{O} 3-\mathrm{S} 3-\mathrm{N} 2$ & $108.14(8)$ & $\mathrm{C} 8-\mathrm{C} 7-\mathrm{H} 7 \mathrm{~B}$ & 109.2 \\
\hline $\mathrm{O} 4-\mathrm{S} 3-\mathrm{C} 3$ & $109.63(8)$ & $\mathrm{H} 7 \mathrm{~A}-\mathrm{C} 7-\mathrm{H} 7 \mathrm{~B}$ & 107.9 \\
\hline $\mathrm{O} 3-\mathrm{S} 3-\mathrm{C} 3$ & $108.34(8)$ & $\mathrm{C} 9-\mathrm{C} 8-\mathrm{C} 7$ & $112.58(16)$ \\
\hline $\mathrm{N} 2-\mathrm{S} 3-\mathrm{C} 3$ & $100.62(7)$ & $\mathrm{C} 9-\mathrm{C} 8-\mathrm{H} 8 \mathrm{~A}$ & 109.1 \\
\hline $\mathrm{C} 10-\mathrm{O} 5-\mathrm{C} 9$ & $114.96(14)$ & $\mathrm{C} 7-\mathrm{C} 8-\mathrm{H} 8 \mathrm{~A}$ & 109.1 \\
\hline $\mathrm{S} 1-\mathrm{N} 1-\mathrm{H} 1 \mathrm{~A}$ & $118.3(14)$ & $\mathrm{C} 9-\mathrm{C} 8-\mathrm{H} 8 \mathrm{~B}$ & 109.1 \\
\hline $\mathrm{S} 1-\mathrm{N} 1-\mathrm{H} 1 \mathrm{~B}$ & $118.6(15)$ & $\mathrm{C} 7-\mathrm{C} 8-\mathrm{H} 8 \mathrm{~B}$ & 109.1 \\
\hline $\mathrm{H} 1 \mathrm{~A}-\mathrm{N} 1-\mathrm{H} 1 \mathrm{~B}$ & $112(2)$ & $\mathrm{H} 8 \mathrm{~A}-\mathrm{C} 8-\mathrm{H} 8 \mathrm{~B}$ & 107.8 \\
\hline $\mathrm{C} 6-\mathrm{N} 2-\mathrm{C} 7$ & $114.80(13)$ & $\mathrm{O} 5-\mathrm{C} 9-\mathrm{C} 8$ & $112.38(16)$ \\
\hline $\mathrm{C} 6-\mathrm{N} 2-\mathrm{S} 3$ & $110.94(10)$ & $\mathrm{O} 5-\mathrm{C} 9-\mathrm{H} 9 \mathrm{~A}$ & 109.1 \\
\hline $\mathrm{C} 7-\mathrm{N} 2-\mathrm{S} 3$ & $116.48(11)$ & $\mathrm{C} 8-\mathrm{C} 9-\mathrm{H} 9 \mathrm{~A}$ & 109.1 \\
\hline $\mathrm{C} 11-\mathrm{N} 3-\mathrm{C} 5$ & $116.46(13)$ & $\mathrm{O} 5-\mathrm{C} 9-\mathrm{H} 9 \mathrm{~B}$ & 109.1 \\
\hline $\mathrm{C} 11-\mathrm{N} 3-\mathrm{H} 3$ & $105.9(14)$ & $\mathrm{C} 8-\mathrm{C} 9-\mathrm{H} 9 \mathrm{~B}$ & 109.1 \\
\hline $\mathrm{C} 5-\mathrm{N} 3-\mathrm{H} 3$ & $106.0(14)$ & $\mathrm{H} 9 \mathrm{~A}-\mathrm{C} 9-\mathrm{H} 9 \mathrm{~B}$ & 107.9 \\
\hline $\mathrm{C} 2-\mathrm{C} 1-\mathrm{S} 2$ & $113.32(10)$ & $\mathrm{O} 5-\mathrm{C} 10-\mathrm{H} 10 \mathrm{~A}$ & 109.5 \\
\hline $\mathrm{C} 2-\mathrm{C} 1-\mathrm{S} 1$ & $126.55(10)$ & $\mathrm{O} 5-\mathrm{C} 10-\mathrm{H} 10 \mathrm{~B}$ & 109.5 \\
\hline $\mathrm{S} 2-\mathrm{C} 1-\mathrm{S} 1$ & $119.95(9)$ & $\mathrm{H} 10 \mathrm{~A}-\mathrm{C} 10-\mathrm{H} 10 \mathrm{~B}$ & 109.5 \\
\hline $\mathrm{C} 1-\mathrm{C} 2-\mathrm{C} 4$ & $112.31(12)$ & $\mathrm{O} 5-\mathrm{C} 10-\mathrm{H} 10 \mathrm{C}$ & 109.5 \\
\hline $\mathrm{C} 1-\mathrm{C} 2-\mathrm{H} 2$ & 123.8 & $\mathrm{H} 10 \mathrm{~A}-\mathrm{C} 10-\mathrm{H} 10 \mathrm{C}$ & 109.5 \\
\hline $\mathrm{C} 4-\mathrm{C} 2-\mathrm{H} 2$ & 123.8 & $\mathrm{H} 10 \mathrm{~B}-\mathrm{C} 10-\mathrm{H} 10 \mathrm{C}$ & 109.5 \\
\hline
\end{tabular}




$\begin{array}{llll}\mathrm{C} 4-\mathrm{C} 3-\mathrm{S} 2 & 113.72(11) & \mathrm{N} 3-\mathrm{C} 11-\mathrm{C} 12 & 111.18(16) \\ \mathrm{C} 4-\mathrm{C} 3-\mathrm{S} 3 & 121.46(12) & \mathrm{N} 3-\mathrm{C} 11-\mathrm{H} 11 \mathrm{~A} & 109.4 \\ \mathrm{~S} 2-\mathrm{C} 3-\mathrm{S} 3 & 124.60(9) & \mathrm{C} 12-\mathrm{C} 11-\mathrm{H} 11 \mathrm{~A} & 109.4 \\ \mathrm{C} 3-\mathrm{C} 4-\mathrm{C} 2 & 110.94(13) & \mathrm{N} 3-\mathrm{C} 11-\mathrm{H} 11 \mathrm{~B} & 109.4 \\ \mathrm{C} 3-\mathrm{C} 4-\mathrm{C} 5 & 125.25(13) & \mathrm{C} 12-\mathrm{C} 11-\mathrm{H} 11 \mathrm{~B} & 108.0 \\ \mathrm{C} 2-\mathrm{C} 4-\mathrm{C} 5 & 123.73(13) & \mathrm{C} 11-\mathrm{C} 12-\mathrm{H} 12 \mathrm{~A} & 109.5 \\ \mathrm{~N} 3-\mathrm{C} 5-\mathrm{C} 4 & 113.21(12) & \mathrm{C} 11-\mathrm{C} 12-\mathrm{H} 12 \mathrm{~B} & 109.5 \\ \mathrm{~N} 3-\mathrm{C} 5-\mathrm{C} 6 & 109.05(12) & \mathrm{H} 12 \mathrm{~A}-\mathrm{C} 12-\mathrm{H} 12 \mathrm{~B} & 109.5 \\ \mathrm{C} 4-\mathrm{C} 5-\mathrm{C} 6 & 112.84(13) & \mathrm{C} 11-\mathrm{C} 12-\mathrm{H} 12 \mathrm{C} & 109.5 \\ \mathrm{~N} 3-\mathrm{C} 5-\mathrm{H} 5 & 107.1 & \mathrm{H} 12 \mathrm{~A}-\mathrm{C} 12-\mathrm{H} 12 \mathrm{C} & 109.5 \\ \mathrm{C} 4-\mathrm{C} 5-\mathrm{H} 5 & 107.1 & & 109.5 \\ \mathrm{C} 6-\mathrm{C} 5-\mathrm{H} 5 & 107.1 & & \\ \mathrm{~N} 2-\mathrm{C} 6-\mathrm{C} 5 & 113.55(12) & & \end{array}$

Hydrogen-bond geometry $\left(A,{ }^{\circ}\right)$

\begin{tabular}{lllll}
\hline$D-\mathrm{H} \cdots A$ & $D-\mathrm{H}$ & $\mathrm{H} \cdots A$ & $D \cdots A$ & $D-\mathrm{H} \cdots A$ \\
\hline $\mathrm{N} 1-\mathrm{H} 1 B \cdots \mathrm{O} 5^{\mathrm{i}}$ & $0.87(1)$ & $1.98(1)$ & $2.841(2)$ & $177(2)$ \\
$\mathrm{N} 1-\mathrm{H} 1 A \cdots \mathrm{N} 3^{\mathrm{ii}}$ & $0.87(1)$ & $2.03(1)$ & $2.886(2)$ & $171(2)$ \\
$\mathrm{N} 3-\mathrm{H} 3 \cdots \mathrm{O} 1^{\mathrm{iii}}$ & $0.86(1)$ & $2.26(1)$ & $3.042(2)$ & $151(2)$ \\
\hline
\end{tabular}

Symmetry codes: (i) $x, y+1, z-1$; (ii) $x, y+1, z$; (iii) $-x, y-1 / 2,-z+1$. 